\title{
Magnetic properties of optimized cobalt nanospheres grown by focused electron beam induced deposition (FEBID) on cantilever tips
}

\author{
Soraya Sangiao*1,2, César Magén ${ }^{1,2,3,4}$, Darius Mofakhami ${ }^{5}$, Grégoire de Loubens ${ }^{5}$ \\ and José María De Teresa ${ }^{1,2,4}$
}

\section{Full Research Paper}

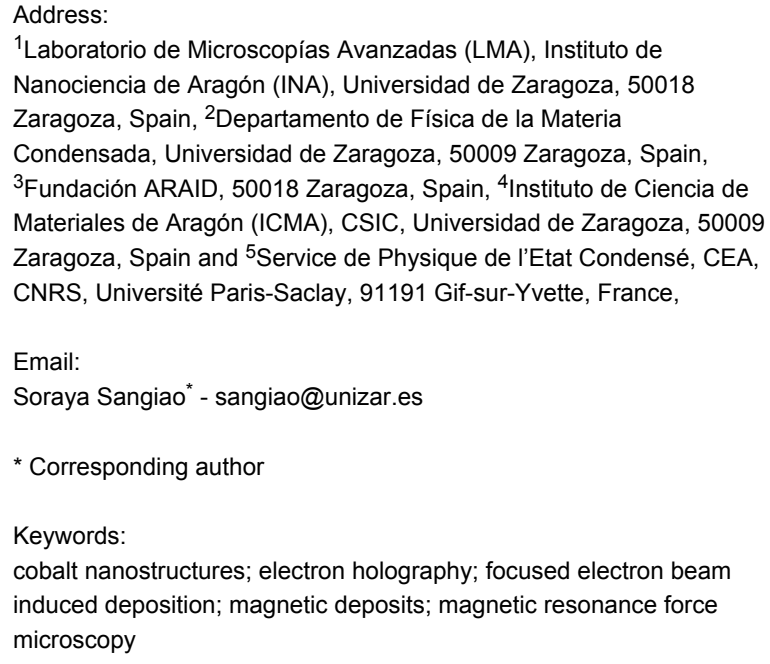

${ }^{1}$ Laboratorio de Microscopías Avanzadas (LMA), Instituto de Nanociencia de Aragón (INA), Universidad de Zaragoza, 50018 Zaragoza, Spain, ${ }^{2}$ Departamento de Física de la Materia Condensada, Universidad de Zaragoza, 50009 Zaragoza, Spain, ${ }^{3}$ Fundación ARAID, 50018 Zaragoza, Spain, ${ }^{4}$ Instituto de Ciencia de Materiales de Aragón (ICMA), CSIC, Universidad de Zaragoza, 50009 Zaragoza, Spain and ${ }^{5}$ Service de Physique de l'Etat Condensé, CEA, CNRS, Université Paris-Saclay, 91191 Gif-sur-Yvette, France,

\section{Email:}

Soraya Sangiao* - sangiao@unizar.es

* Corresponding author

Keywords:

cobalt nanostructures; electron holography; focused electron beam induced deposition; magnetic deposits; magnetic resonance force microscopy

\begin{abstract}
Beilstein J. Nanotechnol. 2017, 8, 2106-2115. doi:10.3762/bjnano.8.210
\end{abstract}

Received: 19 June 2017

Accepted: 12 September 2017

Published: 09 October 2017

This article is part of the Thematic Series "Chemistry for electron-induced nanofabrication".

Guest Editor: H. Marbach

(C) 2017 Sangiao et al.; licensee Beilstein-Institut. License and terms: see end of document.

\footnotetext{
Abstract

In this work, we present a detailed investigation of the magnetic properties of cobalt nanospheres grown on cantilever tips by focused electron beam induced deposition (FEBID). The cantilevers are extremely soft and the cobalt nanospheres are optimized for magnetic resonance force microscopy (MRFM) experiments, which implies that the cobalt nanospheres must be as small as possible while bearing high saturation magnetization. It was found that the cobalt content and the corresponding saturation magnetization of the nanospheres decrease for nanosphere diameters less than $300 \mathrm{~nm}$. Electron holography measurements show the formation of a magnetic vortex state in remanence, which nicely agrees with magnetic hysteresis loops performed by local magnetometry showing negligible remanent magnetization. As investigated by local magnetometry, optimal behavior for high-resolution MRFM has been found for cobalt nanospheres with a diameter of $\approx 200 \mathrm{~nm}$, which present atomic cobalt content of $\approx 83$ atom $\%$ and saturation magnetization of $10^{6} \mathrm{~A} / \mathrm{m}$, around $70 \%$ of the bulk value. These results represent the first comprehensive investigation of the magnetic properties of cobalt nanospheres grown by FEBID for application in MRFM.
} 


\section{Introduction}

Through the local decomposition of magnetic precursor molecules by the action of an incoming electron beam, a wide range of functional magnetic nanostructures have been produced in last years by the focused electron beam induced deposition (FEBID) technique [1,2]. The extensive list of nanostructures includes: (a) planar deposits in the shape of Hall bars for sensing purposes [3-6]; (b) magnetic nanopillars for functionalization of tip cantilevers with applications in magnetic force microscopy (MFM) [7-10] and magnetic resonance force microscopy (MRFM) [11]; (c) planar nanowires for application in magnetic domain-wall conduits $[12,13]$, in logic circuits $[14,15]$, in dense memory arrays [16] and for superconductingvortex-lattice pinning [17]; as well as (d) three-dimensional nanowires for magnetic domain-wall studies $[18,19]$ and for remote magnetomechanical actuation [20], quantum dots for magnetic storage [21] and catalytic purposes [22], polygonal shapes for micromagnetic studies [23,24] and spin-ice investigations [25], nanoconstrictions and nanocontacts for domainwall pinning [26] and Andreev reflection studies [27]. The growth of such numerous types of $2 \mathrm{D}$ and $3 \mathrm{D}$ magnetic nanostructures has been possible thanks to the main virtues of the FEBID technique such as: arbitrary design of the beam scan path [28], high resolution provided by the fine electron beam spot [29], tuning of growth parameters (beam dwell time, precursor flux, etc.) $[30,31]$ and flexibility in the type of substrate used (rigid or flexible, flat or curved, conductive or insulating) [32].

An important aspect to consider in the growth by FEBID is the metal content, which is generally linked to the functionality of the deposit. In the case of magnetic deposits grown by FEBID, the metal content can be finely tuned in various ways. The beam current [7,33], the beam dwell time [30], the precursor flux [5], the beam voltage [34] and the substrate temperature $[35,36]$ have been found to be relevant parameters to tune the metal content in magnetic deposits. However, some constraints exist, which impede to grow arbitrary shapes with arbitrary metal content. In general, the difficulties increase when the goal is to grow very small structures (smaller than $100 \mathrm{~nm}$ ) with high metal content. Another strategy to increase the metal content and/or change the microstructure arises in the application of post-growth purification steps [37-39]. In order to avoid the sur- face oxidation of the magnetic nanostructures, the use of protective shells has been found to be very effective $[31,40]$.

In the present work, we extend the growth of cobalt nanospheres using FEBID for application in MRFM. MRFM is a quantitative magnetic characterization technique that exploits the tiny magnetic forces appearing between a magnetic tip and a magnetic sample for the investigation of spin dynamics at the nanoscale [41]. This near field scanning probe technique allows magnetic resonance imaging (MRI) with nanometer spatial resolution and extreme spin sensitivity [42] and the investigation of spin waves at the sub-micrometer scale [43-45]. In these applications, very strong field gradients from the magnetic probe [46] and ultra-soft cantilevers [47] are required. Therefore, the magnetic probe should be precisely located at the apex of the cantilever and be as small as possible to gain spatial resolution, and it should have as high magnetization as possible to maximize the MRFM signal [48]. Moreover, a spherical shape is beneficial to minimize hysteresis effects and makes quantitative analysis more easy [49]. These requirements imply the optimization of the FEBID growth in order to obtain cobalt spheres sufficiently small but at the same time having high metal content in order to present high saturation magnetization.

\section{Results and Discussion Sample growth and characterization}

In FEBID, the precursor gas molecules are delivered onto the substrate surface by means of a nearby gas-injection system and the focused electron beam is scanned on the surface. The precursor gas molecules are dissociated by electron beam irradiation, creating a deposit with the same shape of the scanning beam. The cobalt nanospheres were grown by FEBID under $5 \mathrm{kV}$ electron beam voltage. For cobalt nanospheres with a diameter greater than $150 \mathrm{~nm}$, an electron beam current of $1.6 \mathrm{nA}$ was used, whereas for growing smaller nanospheres, an electron beam current of $0.4 \mathrm{nA}$ was chosen. The main growth parameters used for the cobalt nanospheres reported here are listed in Table 1.

In order to synthesize nanospheres, we have taken advantage of the point-like nature of the growth surface, that is, the apex of the cantilever (Olympus BioLever, around $30 \mathrm{~nm}$ in size). We

Table 1: Growth parameters used for the cobalt nanospheres in the present study. In the last column, the cobalt content (atomic percent) of a cobalt nanosphere of $400 \mathrm{~nm}$ in diameter grown under the reported conditions is given with the measurement precision in parentheses.

\begin{tabular}{llll}
$\Phi$, Nanosphere diameter $(\mathrm{nm})$ & $V_{\text {beam }}(\mathrm{kV})$ & $I_{\text {beam }}(\mathrm{nA})$ & Co atom \% (for $\Phi=400 \mathrm{~nm})$ \\
\hline$<150 \mathrm{~nm}$ & 5.0 & 0.4 & $93(2)$ \\
$\Phi \geq 150 \mathrm{~nm}$ & 5.0 & 1.6 & $91(2)$
\end{tabular}


have scanned the beam over a circular area centered on the apex of the cantilevers and varied the radius of the circular area being scanned and the beam scanning time to obtain the different targeted diameters. The diameter of the circular area is constant during the growth of each nanosphere and equal to approximately $75 \%$ of the targeted diameter. The beam shift, together with live imaging, were used to ensure that the circular area being scanned is always centered on the apex of the cantilever. From several optimization experiments, we have chosen the optimal radius and time of the circular area being scanned for growing nanospheres with desired diameters. As shown in Figure 1 for three different cobalt nanospheres grown by FEBID, we are able to fabricate cobalt nanospheres with the desired diameter by optimizing the radius and the circular area scan time on the apex of the cantilever. The nanospheres required growth times ranging from 2 to $6 \mathrm{~s}$. In the present work we have fabricated cobalt nanospheres with diameters ranging from $500 \mathrm{~nm}$ down to $90 \mathrm{~nm}$ with good spherical geometry and a smooth surface.

We have studied the cobalt content of the nanospheres grown by FEBID at the apex of cantilevers by energy dispersive X-ray spectroscopy (EDS) to check the evolution of the metal content with nanosphere diameter, as it changes the surface-to-volume ratio of the nanosphere. First, we have grown nanospheres of $400 \mathrm{~nm}$ in diameter using the two different sets of growth parameters reported in Table 1, which are the most appropriate for growing cobalt nanospheres with diameters either less than or

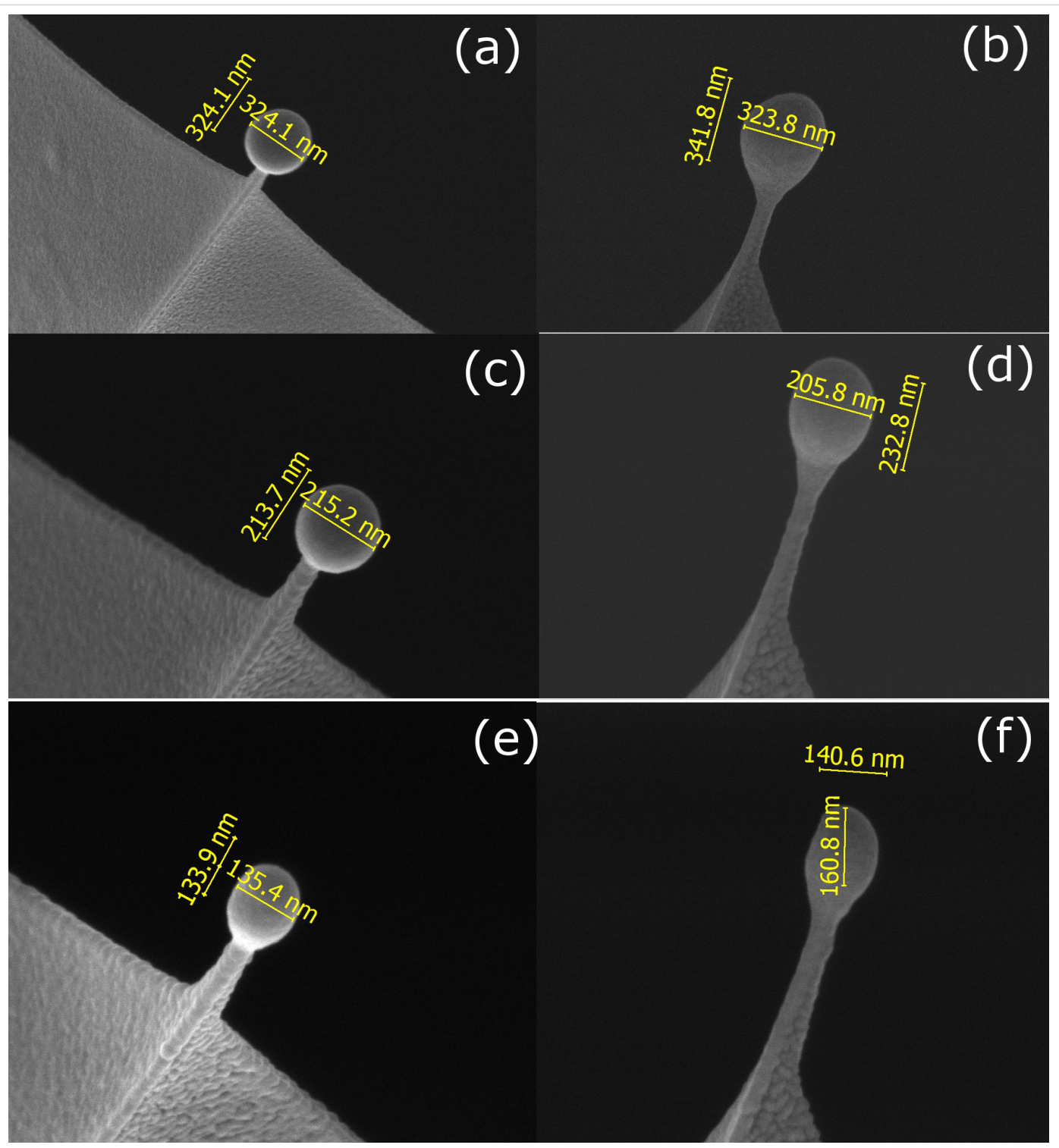

Figure 1: SEM micrographs showing the dimensions of the grown cobalt nanospheres. Top view and front view of cobalt nanospheres of $325 \mathrm{~nm}$ $(\mathrm{a}, \mathrm{b}), 215 \mathrm{~nm}(\mathrm{c}, \mathrm{d})$ and $135 \mathrm{~nm}(\mathrm{e}, \mathrm{f})$ in diameter. 
greater than $150 \mathrm{~nm}$, respectively. The obtained cobalt content for the nanospheres grown as listed in the last column of Table 1 are very similar to each other with values of 93 atom \% Co and 91 atom \% Co, which constitutes a difference that is below the experimental accuracy. As shown in Figure 2, the cobalt content, in atomic percent, decreases as the diameter of the nanosphere decreases, down to the minimum value found of 60 atom \% Co for the smallest nanosphere of $90 \mathrm{~nm}$ in diameter. The optimized cobalt content, $\approx 92$ atom $\%$, is obtained only for nanospheres with diameter greater than $400 \mathrm{~nm}$. We attribute the decrease in the cobalt content for diameters less than $400 \mathrm{~nm}$ to the natural surface oxidation of the cobalt nanospheres, which occurs in a spherical shell with an outer radius equal to the radius of the particular nanosphere and a thickness of approximately $5 \mathrm{~nm}$. Another explanation, besides native surface oxidation, for the decrease in cobalt content for diameters less than $400 \mathrm{~nm}$ could be a change in the growth mode, as previously reported in 3D cobalt nanowires grown by FEBID [31]. In $3 \mathrm{D}$ cobalt nanowires the growth occurs in a radial mode for a wire diameter greater than $120 \mathrm{~nm}$, which results in higher Co content than wires with a diameter less than $80 \mathrm{~nm}$, which grow in a linear mode.

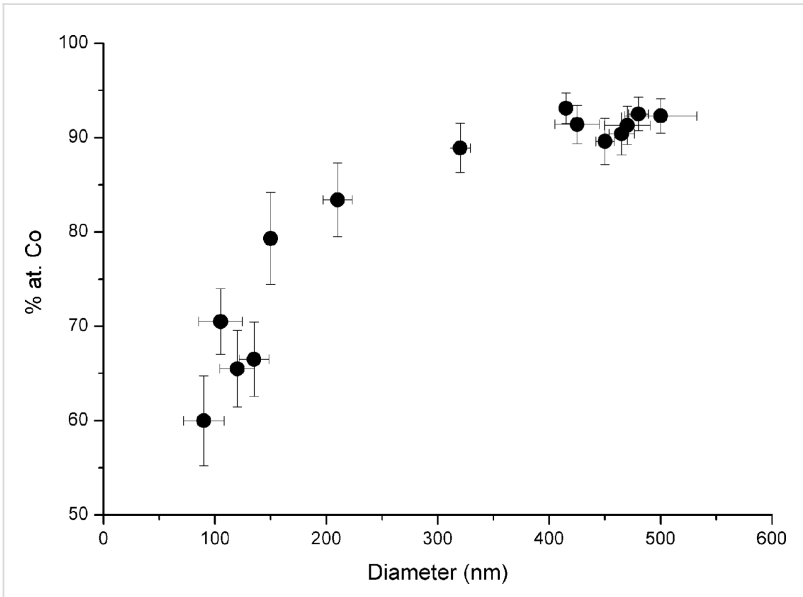

Figure 2: Composition of the cobalt nanospheres, as measured by EDX, as a function of diameter.

In order to analyze the chemical composition of the cobalt nanospheres by electron energy loss spectroscopy (EELS) in scanning transmission electron microscopy (STEM) mode and their local magnetic properties by electron holography in a transmission electron microscope (TEM), the specimens were prepared for TEM observation in a specific geometry. Firstly, the cantilever pyramid tip is cut by focused ion beam (FIB) milling and lifted-out by a micromanipulator. Then, the cantilever tip is welded onto a TEM copper grid by a FIB-induced Pt deposition, as illustrated in Figure 3a. Then, the FEBID cobalt nanosphere is grown at the apex of the cantilever, following the same procedure as described above. Figure 3b,c displays the SEM micrographs of the two cobalt nanospheres studied by STEM-EELS and electron holography, once grown at the apex of cantilevers already attached to the TEM grid.

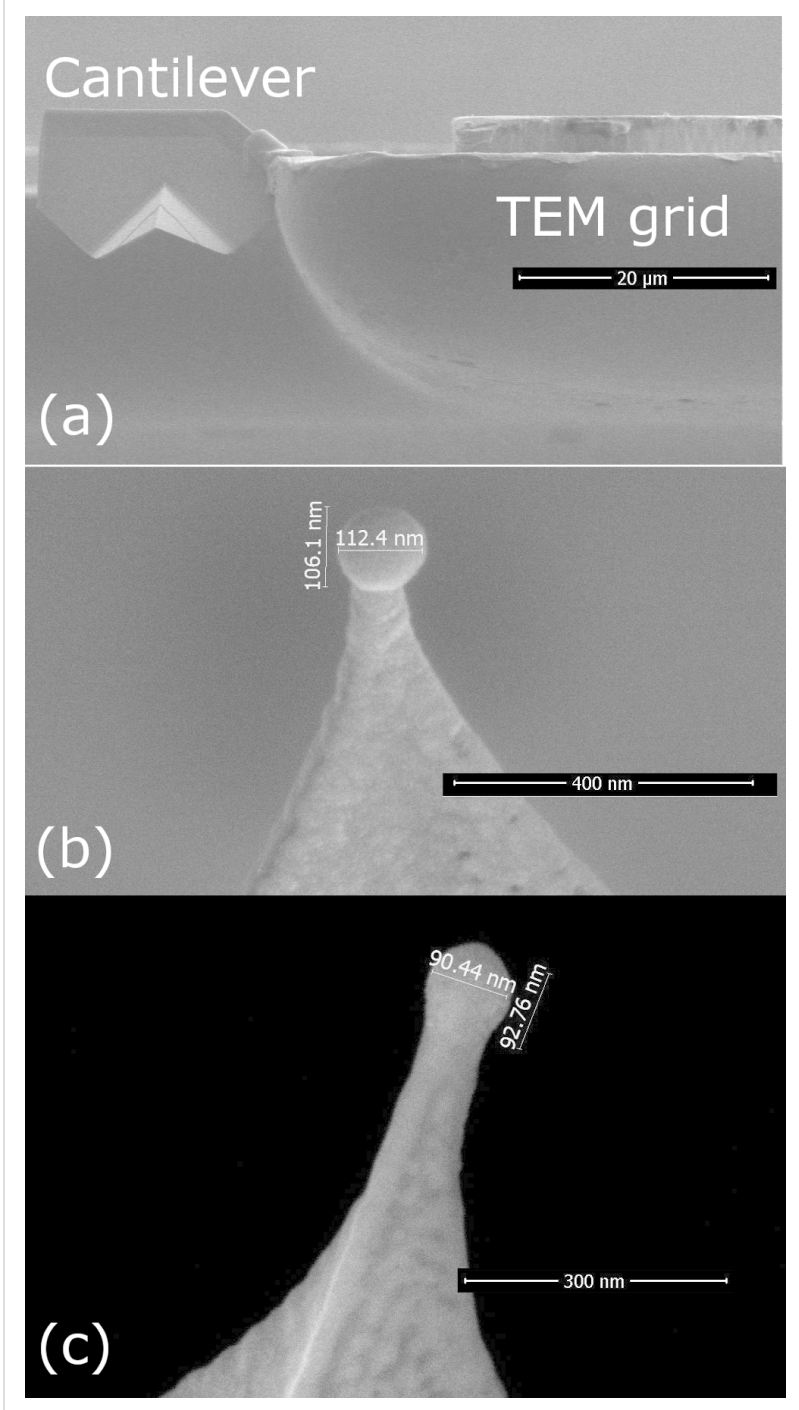

Figure 3: SEM micrographs of the cobalt nanospheres grown on cantilever tips for STEM-EELS and electron holography experiments. (a) Cantilever pyramid tip welded to a TEM grid. (b,c) Cobalt nanospheres grown by FEBID on the apex of the cantilever already attached to the TEM grid. The diameter of the nanospheres shown is $110 \mathrm{~nm}$ (b) and $90 \mathrm{~nm}(\mathrm{c})$.

The morphological and compositional properties of the cobalt nanospheres grown by FEBID have been confirmed by local chemical mapping of selected nanospheres of diameters $110 \mathrm{~nm}$ (see Figure 4b) and $90 \mathrm{~nm}$ (see Supporting Information File 1) performed by STEM-EELS. These quantitative maps reveal, first of all, that the deposits are not perfect spheres attached to the tip. On the other hand, they appear to be partially stuck into the tip of the cantilever, in particular the smallest sphere. For 
this morphology, secondary electrons that cause the decomposition of the precursor are emitted all around the tip; thus, at the early stages of the growth, cobalt atoms wrap around the tip of the pyramid. A colored chemical map, including the relative compositions of Co (red), O (green) and C (blue), the only chemical elements detected in the nanospheres, is shown in Figure $4 \mathrm{~b}$ for the nanosphere of $110 \mathrm{~nm}$ in diameter. This chemical map can be analyzed quantitatively, as displayed in Figure $4 \mathrm{c}$, obtaining a net Co content at the center of the nanosphere of about 80 atom $\%$ with respect to the total composition of $\mathrm{Co}, \mathrm{C}$ and $\mathrm{O}$. A remarkable oxidation layer is observed, extending approximately $6 \mathrm{~nm}$. This agrees nicely with previous reports on Co-FEBID, which have confirmed this layer to be non-ferromagnetic [50]. Furthermore, a thin layer containing carbon and oxygen of about $7 \mathrm{~nm}$ is formed due to contamination before and during the electron beam irradiation in the TEM experiment. As a result, the average diameter of cobalt under the oxidation layer and possible contaminant extends to $100 \mathrm{~nm}$ out of the $110 \mathrm{~nm}$ of the whole sphere diameter.
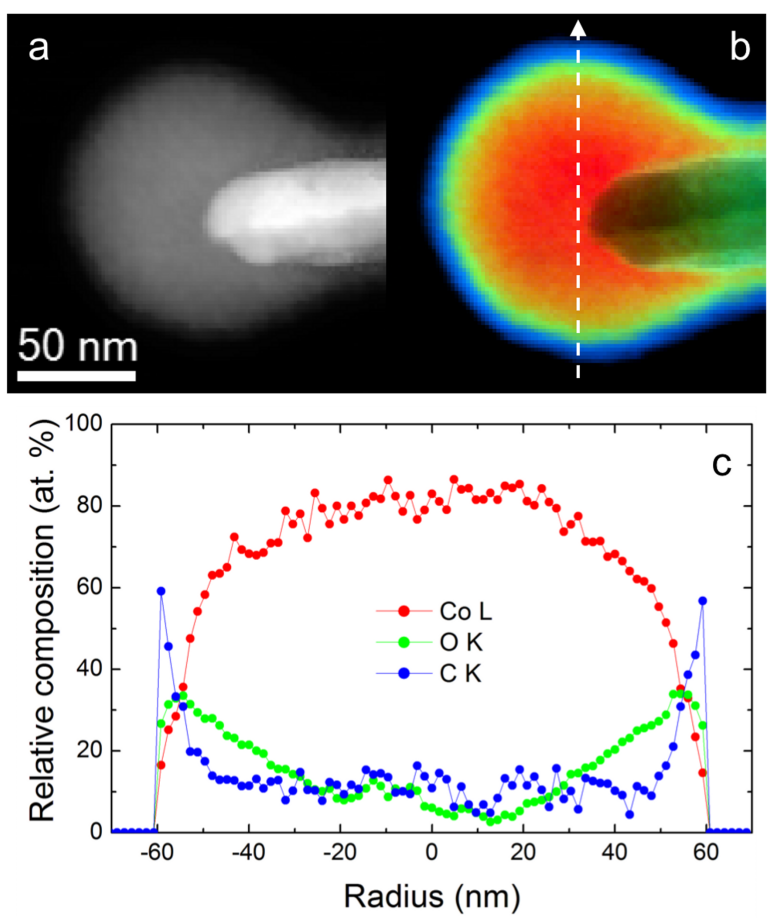

Figure 4: STEM-EELS compositional analysis of the cobalt nanosphere with $110 \mathrm{~nm}$ diameter. (a) Reference image in Z contrast. (b) Colored chemical map, including the relative compositions of Co (red), O (green) and C (blue). (c) Compositional line profile extracted along the white arrow in (b).

\section{Local magnetic characterization by electron holography}

The remanent magnetic state of the two Co nanospheres with approximately $110 \mathrm{~nm}$ and $90 \mathrm{~nm}$ diameter (see Figure 3b,c) has been imaged by off-axis electron holography in a TEM [51].

Figure 5 illustrates the hologram acquisition and retrieval of the magnetic induction flux distribution in the nanosphere with $110 \mathrm{~nm}$ grown on the cantilever tips (see the analysis of the nanosphere with $90 \mathrm{~nm}$ diameter in Supporting Information File 1). Figure 5a shows the bright field image of the Co nanosphere overlapped with the interference fringe pattern of the hologram, revealing a significant amount of contamination which did not disappear after standard $\mathrm{Ar} / \mathrm{O}_{2}$ plasma cleaning procedures. Furthermore, the holograms before and after (not shown) reversing the object show how contamination builds up during the experiment, which affects the quantitative properties of the technique (particularly for the smaller sphere of $90 \mathrm{~nm}$, shown in the Supporting Information File 1).

The electrostatic $\left(\varphi_{E}\right)$ and magnetic $\left(\varphi_{M}\right)$ contributions to the phase shift retrieved from the hologram analysis are shown in Figure $5 \mathrm{~b}$ and Figure $5 \mathrm{c}$, respectively. In particular, the magnetic contribution illustrated in Figure $5 \mathrm{c}$ is shown in terms of $\cos \left(12 \cdot \varphi_{\mathrm{M}}\right)$, giving rise to a fringe pattern that corresponds to the distribution of magnetic induction flux lines produced by the magnetic object. As a result, the nanosphere presents a nearly circular closure domain of magnetic induction, circulating counterclockwise, with the in-plane magnetization decreasing while approaching the center of the nanosphere. No in-plane stray fields are observed. This magnetic induction geometry corresponds to a counterclockwise vortex state of undetermined polarity (it is compatible with the magnetic flux leaking at the center of the sphere both into or out of the image plane). Quantitative values of the in-plane magnetic induction can be extracted by estimating the local thickness of the sample using the electrostatic phase image. This is done by assuming that the area of maximum phase around the center of the sphere corresponds to a nominal thickness of $110 \mathrm{~nm}$ (the contamination layer is ignored, assuming that its contribution to the average mean inner potential of the object is reduced). Using this "thickness" image, the absolute in-plane magnetic induction map can be determined. A line profile of the net in-plane magnetic induction distribution along the white arrow in Figure $5 \mathrm{c}$ is displayed in Figure 5d. This magnetic induction profile matches again that of a vortex state in which the maximum magnetic induction values are observed at the outer regions of there; these values diminish while approaching the center of the sphere due to the rotation of the magnetization out-of-plane until the in-plane magnetic induction is nearly zero around the center of the nanosphere, which corresponds to the vortex core. The maximum value of the magnetic induction is approximately $1.1 \pm 0.1 \mathrm{~T}$, which agrees nicely with previous magnetization values determined for similar nanodeposits, such as 

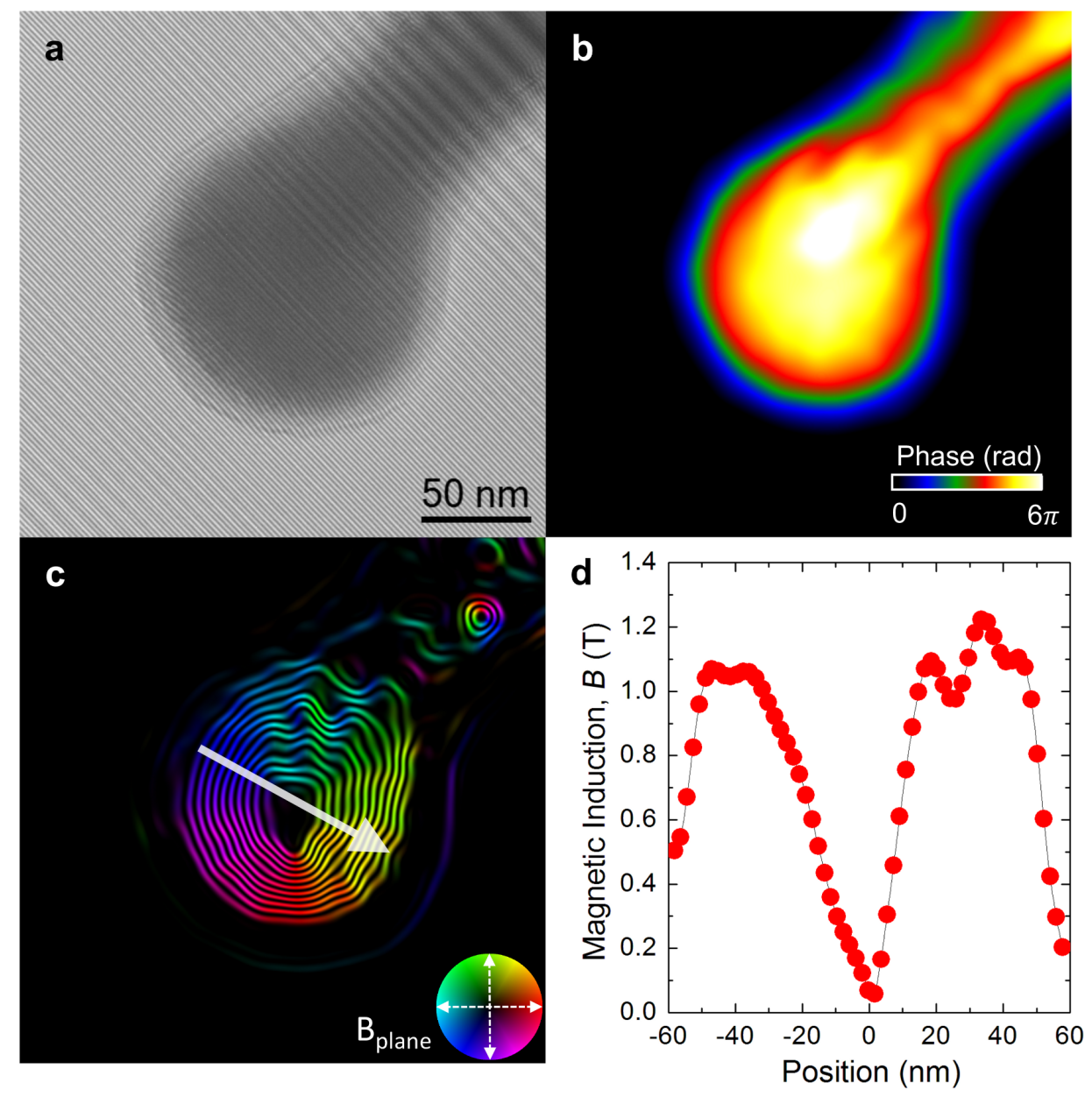

Figure 5: Electron holography of one cobalt nanosphere with a diameter of $110 \mathrm{~nm}$. a) Electron hologram of the object. b) Electrostatic phase image, $\varphi_{E}$. c) Colored representation of the in-plane magnetic induction flux lines, represented as $\cos \left(12 \cdot \varphi_{\mathrm{M}}\right)$. The inset represents the color scale of the magnetic induction orientation in arbitrary units, where the position of a color relative to the center of the circle corresponds to the orientation of the magnetic induction. d) Profile of the in-plane component of the magnetic induction vector as measured along the white arrow $\mathrm{c}$ ), where the position reference is taken at the minimum of the magnetic induction.

vertical nanowires [31]. In both cases, the deposition condition gives rise to magnetization values reduced with respect to bulk values due to the moderate purity of the deposit and the reduction of the effective magnetic volume due to the formation of a non-magnetic oxide surface layer [50].

\section{Magnetization measurements of the cobalt nanospheres}

In order to measure the magnetization of the cobalt nanospheres, we take advantage of them being attached at the end of very sensitive force sensors to perform cantilever magnetometry. The mechanical resonance frequency of the cantilever is monitored as a function of the applied magnetic field while it is positioned in a strong field gradient created by a cylindrical magnetic microwire [11] (see inset of Figure 6a and the Experimental section for details on the setup). Due to the low stiffness (spring constant $k=6 \mathrm{mN} / \mathrm{m}$ ) and high quality factor (2000<Q<4000 under vacuum) of the cantilever, its frequency accurately probes the magnetic force produced by the field gradient on the nanosphere. In the experimental conditions, the cantilever frequency shift is directly proportional to the magnetization of the cobalt nanosphere (see Equation 1 in the Experimental section), which allows simple extraction of its hysteresis curve. This is shown in Figure 6a,b for a cobalt nanosphere having a diameter of $500 \mathrm{~nm}$. The nanosphere is fully saturated above $0.6 \mathrm{~T}$, and its magnetization decreases quite linearly with the field below this value to become negligible in the remanent state. These magnetometry data also allow us to quantitatively extract the magnetization of the nanosphere [11]. From the maximal relative variation of the cantilever frequency $(1.2 \%$ in Figure 6a) and knowing the cantilever spring constant and the second spatial derivative of the magnetic field 

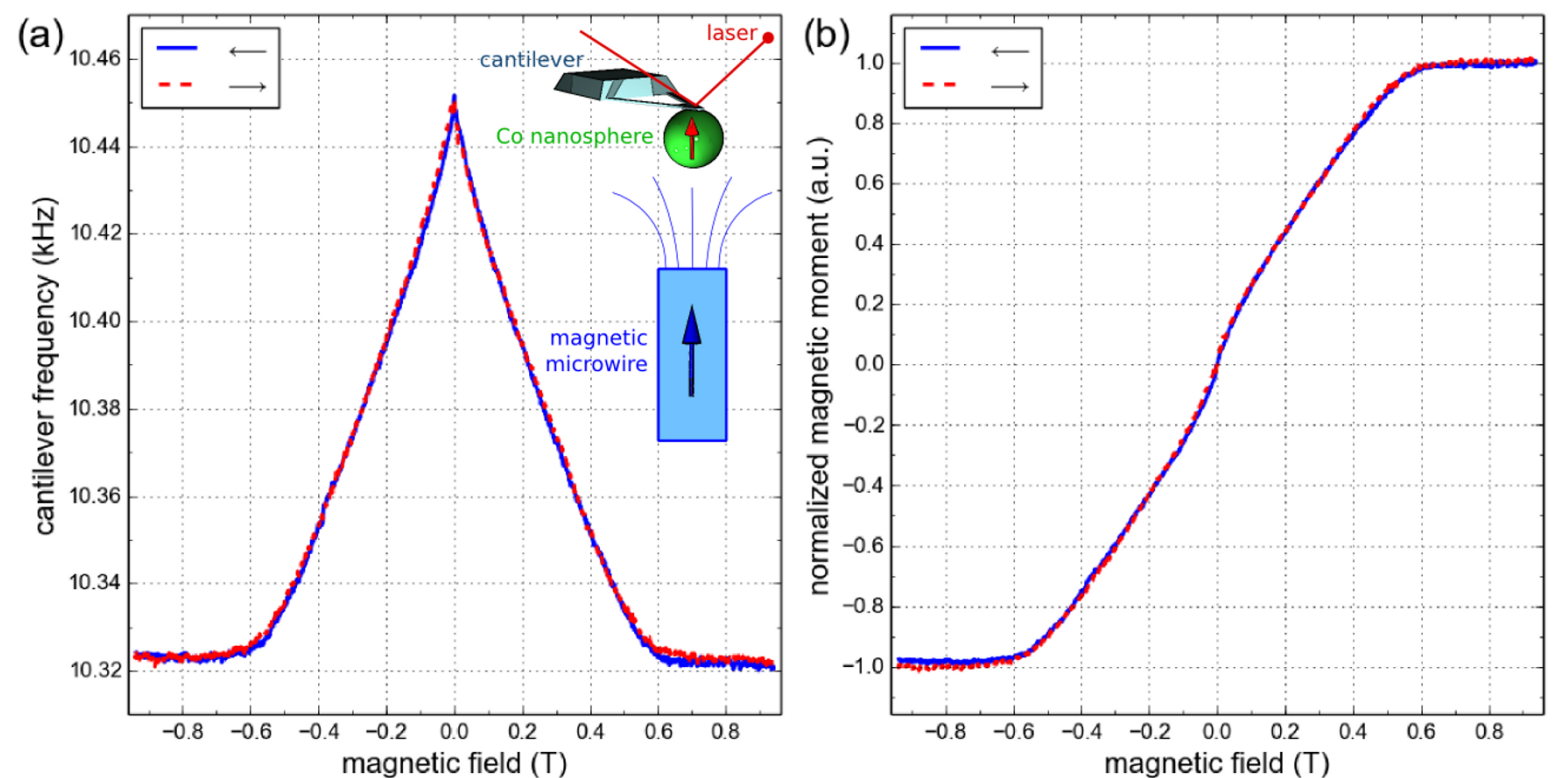

Figure 6: Cantilever magnetometry of a $500 \mathrm{~nm}$ diameter cobalt nanosphere. (a) Raw data of the cantilever frequency vs applied magnetic field. Inset: Sketch of the cantilever magnetometry setup. (b) Extracted magnetization curve.

$\left((1.5 \pm 0.3) \times 10^{9} \mathrm{~T} / \mathrm{m}^{2}\right)$ in which the measurements are operated, one can estimate the magnetic moment of the $500 \mathrm{~nm}$ diameter cobalt nanosphere to be $(1 \pm 0.2) \times 10^{-13} \mathrm{~A} \cdot \mathrm{m}^{2}$. Divided by the volume of the nanosphere, this yields a saturation magnetization $M_{\mathrm{S}}=1450 \pm 300 \mathrm{kA} / \mathrm{m}$, which compares well to the bulk value of cobalt at room temperature $(1400 \mathrm{kA} / \mathrm{m})$. To check the consistency of this estimate and obtain a better accuracy, one can also use the value of the saturation field of the nanosphere. For a perfect sphere without crystalline anisotropy, it is only governed by demagnetizing effects and equal to $\mu_{0} M_{\mathrm{s}} / 3$. This saturation field is accurately determined from a series of measurement similar to the one presented in Figure $6 \mathrm{a}$ by varying the distance from the source of the field gradient. The saturation field for the $500 \mathrm{~nm}$ diameter cobalt nanosphere is found to be $0.58 \pm 0.01 \mathrm{~T}$, which yields $M_{\mathrm{s}}=1385 \pm 25 \mathrm{kA} / \mathrm{m}$ assuming a perfect spherical shape, in very good agreement with the previous estimate.

We have repeated these magnetometry measurements for different magnetic nanospheres of varying diameters. The experimental results are reported in Figure 7, where the dependence of the saturation magnetization upon the diameter of the cobalt nanosphere is displayed. It is found that for diameters larger than $300 \mathrm{~nm}$, the saturation magnetization of the nanosphere is close to bulk cobalt, in good correspondence with the behavior of the cobalt content, which remains close to 90 atom $\%$ in this particle size range. Below $300 \mathrm{~nm}$, the saturation magnetization of the nanosphere quickly drops, similar to the decrease of the cobalt content observed in Figure 2. By extrapolating, one would find that the saturation magnetization vanishes for cobalt content below 50 atom \%. Interestingly, $200 \mathrm{~nm}$ diameter nanospheres still have a magnetization of about $1000 \mathrm{kA} / \mathrm{m}$, which for MRFM application represents the best compromise between spatial resolution and sensitivity.

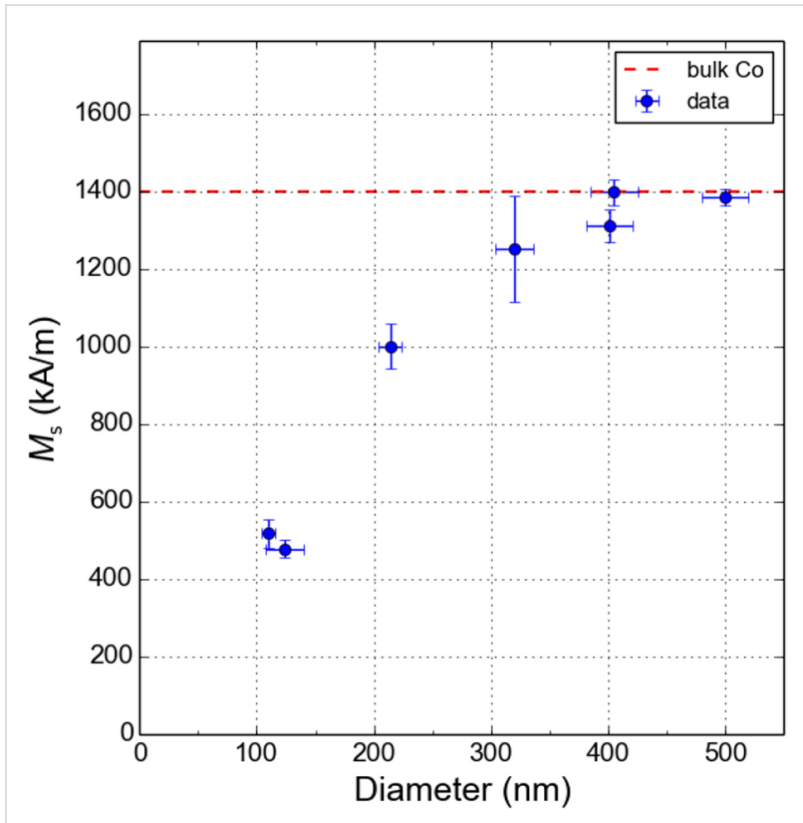

Figure 7: Saturation magnetization of the cobalt nanospheres as a function of their diameter. 


\section{Conclusion}

We have presented here a comprehensive characterization of the chemical and magnetic properties of cobalt nanospheres grown on the apex of a MRFM cantilever by FEBID. EDS analysis of the atomic Co content shows a maximum of 94 atom $\%$ for nanospheres with diameters larger than $400 \mathrm{~nm}$ and a decrease in the Co content for smaller nanospheres.

Quantitative chemical composition analysis by STEM-EELS on a cobalt nanosphere of $110 \mathrm{~nm}$ in diameter showed a relative Co content of 80 atom $\%$ and has revealed the presence of a native oxidation spherical shell of $6 \mathrm{~nm}$ in thickness. Precise characterization of the remanent magnetic state has been performed by electron holography on the cobalt nanospheres of $110 \mathrm{~nm}$ in diameter. The in-plane magnetic induction geometry corresponds to a counterclockwise vortex state.

As investigated by local magnetometry, optimal behavior for high-resolution MRFM has been found for cobalt nanospheres with diameters around $200 \mathrm{~nm}$, which present an atomic cobalt content of 83 atom $\%$ and saturation magnetization of about $10^{6} \mathrm{~A} / \mathrm{m}, 70 \%$ of the cobalt bulk value. This study constitutes the first detailed characterization of the magnetic properties of cobalt nanospheres grown by FEBID for application in MRFM experiments.

\section{Experimental}

Samples have been grown by FEBID using the following parameters: $V_{\text {beam }}=5 \mathrm{kV}, I_{\text {beam }}=0.4 \mathrm{nA}$ for diameter less than $150 \mathrm{~nm}$ and $1.6 \mathrm{nA}$ for diameters greater than $150 \mathrm{~nm}$, beam spot diameter $=8.8 \mathrm{~nm}(0.4 \mathrm{nA}) / 17.6 \mathrm{~nm}(1.6 \mathrm{nA})$, precursor temperature $=27^{\circ} \mathrm{C}$, chamber base pressure $\approx 1.2 \times 10^{-6} \mathrm{mbar}$, chamber growth pressure $\approx 3.5 \times 10^{-6}$ mbar. EDS experiments have been performed using a beam voltage of $5 \mathrm{kV}$.

Cantilever magnetometry measurements were performed at room temperature using the setup described in $[11,49]$. The source of the field gradient is a millimeter long, $16 \mu \mathrm{m}$ diameter cylinder of $\mathrm{CoFeNiSiB}$ alloy, with a saturation magnetization of $510 \mathrm{kA} / \mathrm{m}$. The cobalt nanosphere is positioned at a distance between $5 \mu \mathrm{m}$ and $20 \mu \mathrm{m}$ from the top surface of the cylinder to perform the measurements. A standard laser deflection technique is used to monitor the displacement of the cantilever. Its resonance frequency is tracked using a piezoelectric bimorph and a feedback electronic circuit based on a phase lock loop. The relative frequency shift due to the force acting on the magnetic moment $m$ of the cobalt particle is:

$$
\frac{\Delta f_{\mathrm{c}}}{f_{\mathrm{c}}}=-\frac{m}{2 k}\left(\frac{\partial^{2} B_{z}}{\partial z^{2}}\right)_{\mid z_{0}}
$$

where $k$ is the cantilever spring constant, $B_{z}$ the vertical component of the magnetic field from the cylinder, and $z_{0}$ is the equilibrium position of the particle in the field gradient.

STEM-EELS chemical mapping and quantification was carried out at an acceleration voltage of $300 \mathrm{kV}$ in a probe-corrected FEI Titan 60-300 equipped with a high brightness field emission gun (X-FEG), a CEOS corrector for the condenser system and a Gatan Tridiem 866 ERS image filter/spectrometer. The EELS acquisition was performed with a convergence angle of $25 \mathrm{mrad}$, a collection semi-angle of around $60 \mathrm{mrad}$, an estimated beam current of $160 \mathrm{pA}$ and an exposure time of $30 \mathrm{~ms} /$ pixel. The chemical composition was determined by the standard method of integrated intensity elemental ratios implemented in Gatan's Digital Micrograph software package, using the carbon $\mathrm{K}$, oxygen $\mathrm{K}$ and cobalt $\mathrm{L}_{2,3}$ edges. No further correction for thickness effects was applied.

Off-axis electron holography has been carried out in an FEI Titan Cube 60-300 equipped with a Schottky field emission gun (S-FEG), a CEOS corrector for the objective lens and a motorized electrostatic biprism. The experiments have been performed in aberration-corrected Lorentz mode, with the objective lens switched off and the corrector aligned to compensate the spherical aberration of the Lorentz lens and achieve a spatial resolution of around $1 \mathrm{~nm}$. Electron holograms of $\approx 20 \%$ contrast have been obtained with a biprism excitation of $160 \mathrm{~V}$, an overlap region of about $500 \mathrm{~nm}$ and an acquisition time of $8 \mathrm{~s}$. The electrostatic phase shift $\left(\varphi_{\mathrm{E}}\right)$ and the magnetic phase shift $\left(\varphi_{\mathrm{M}}\right)$ are retrieved by recording two holograms for each object, the second one with the object flipped with respect to the original orientation. In this way, the magnetic contribution contained in the holograms changes sign, while the electrostatic contribution due to the mean inner potential remains unchanged. Once the phases are extracted from both holograms, their subtraction produces a pure magnetic phase shift image and the magnetic induction can be calculated as:

$$
B_{x(y)}=-\frac{\hbar}{e \cdot t} \frac{\partial \varphi_{\mathrm{M}}}{\partial y(x)},
$$

where $\hbar$ is the reduced Planck's constant, $e$ is the electron charge and $t$ is the thickness of the sample.

Visualization of the magnetic state of the Co nanospheres is performed by calculating the cosine of a multiple of the magnetic phase shift, $\cos \left(n \cdot \varphi_{M}\right)$, which produces sets of fringes parallel to the magnetic induction flux. Absolute values of magnetic induction are calculated by estimating the local thickness of the 
object from normalization of $\varphi_{E}$ to the total diameter of the nanosphere.

\section{Supporting Information}

\section{Supporting Information File 1}

Additional experimental information.

Chemical compositional maps from STEM-EELS and

electron holography experiments have been performed on a

$90 \mathrm{~nm}$ diameter sphere following the procedure described

in the main text. These results are described and illustrated in this file.

[http://www.beilstein-journals.org/bjnano/content/ supplementary/2190-4286-8-210-S1.pdf]

\section{Acknowledgements}

This work was supported by Spanish Ministry of Economy and Competitivity through projects No. MAT2014-51982-C2-1-R, MAT2014-51982-C2-2-R and MAT2015-69725-REDT (including FEDER funds), by the Aragon Regional Government, and by EU (Cost Action CM1301- CELINA and ESTEEM2 projects). Experimental help in the sample growth by Laura Casado (LMA-INA) is acknowledged.

\section{References}

1. De Teresa, J. M.; Fernández-Pacheco, A. Appl. Phys. A 2014, 117, 1645-1658. doi:10.1007/s00339-014-8617-7

2. De Teresa, J. M.; Fernández-Pacheco, A.; Córdoba, R.; Serrano-Ramón, L.; Sangiao, S.; Ibarra, M. R. J. Phys. D: Appl. Phys. 2016, 49, 243003. doi:10.1088/0022-3727/49/24/243003

3. Boero, G.; Utke, I.; Bret, T.; Quack, N.; Todorova, M.; Mouaziz, S.; Kejik, P.; Brugger, J.; Popovic, R. S.; Hoffmann, P. Appl. Phys. Lett. 2005, 86, 042503. doi:10.1063/1.1856134

4. Gabureac, M.; Bernau, L.; Utke, I.; Boero, G. Nanotechnology 2010, 21, 115503. doi:10.1088/0957-4484/21/11/115503

5. Serrano-Ramón, L.; Córdoba, R.; Rodríguez, L. A.; Magén, C.; Snoeck, E.; Gatel, C.; Serrano, I.; Ibarra, M. R.; De Teresa, J. M. ACS Nano 2011, 5, 7781-7787. doi:10.1021/nn201517r

6. Córdoba, R.; Lavrijsen, R.; Fernández-Pacheco, A.; Ibarra, M. R.; Schoenaker, F.; Ellis, T.; Barcones-Campo, B.; Kohlhepp, J. T.; Swagten, H. J. M.; Koopmans, B.; Mulders, J. J. L.; De Teresa, J. M. J. Phys. D: Appl. Phys. 2012, 45, 035001. doi:10.1088/0022-3727/45/3/035001

7. Lau, Y. M.; Chee, P. C.; Thong, J. T. L.; Ng, V. J. Vac. Sci. Technol., A 2002, 20, 1295. doi:10.1116/1.1481040

8. Utke, I.; Hoffmann, P.; Berger, R.; Scandella, L. Appl. Phys. Lett. 2002, 80, 4792-4794. doi:10.1063/1.1489097

9. Gavagnin, M.; Wanzenboeck, H. D.; Wachter, S.; Shawrav, M. M.; Persson, A.; Gunnarsson, K.; Svedlindh, P.; Stöger-Pollach, M.; Bertagnolli, E. ACS Appl. Mater. Interfaces 2014, 6, 20254-20260. doi:10.1021/am505785t

10. Belova, L. M.; Hellwig, O.; Dobisz, E.; Dahlberg, E. D. Rev. Sci. Instrum. 2012, 83, 093711. doi:10.1063/1.4752225
11. Lavenant, H.; Naletov, V.; Klein, O.; de Loubens, G.; Casado, L.; De Teresa, J. M. Nanofabrication 2014, 1, 65-73. doi:10.2478/nanofab-2014-0006

12. Fernández-Pacheco, A.; De Teresa, J. M.; Córdoba, R.; Ibarra, M. R.; Petit, D.; Read, D. E.; O'Brien, L.; Lewis, E. R.; Zeng, H. T.; Cowburn, R. P. Appl. Phys. Lett. 2009, 94, 192509. doi:10.1063/1.3139068

13. Idigoras, O.; Nikulina, E.; Porro, J. M.; Vavassori, P.; Chuvilin, A.; Berger, A. Nanofabrication 2014, 1, 23-34. doi:10.2478/nanofab-2014-0003

14. Gavagnin, M.; Wanzenboeck, H. D.; Belić, D.; Bertagnolli, E. ACS Nano 2013, 7, 777-784. doi:10.1021/nn305079a

15. Sharma, N.; van Mourik, R. A.; Yin, Y.; Koopmans, B.; Parkin, S. S. P. Nanotechnology 2016, 27, 165301. doi:10.1088/0957-4484/27/16/165301

16. De Teresa, J. M.; Córdoba, R. ACS Nano 2014, 8, 3788-3795. doi:10.1021/nn500525k

17. Dobrovolskiy, O. V.; Begun, E.; Huth, M.; Shklovskij, V. A.; Tsindlekht, M. I. Physica C 2011, 471, 449-452. doi:10.1016/j.physc.2011.05.245

18. Fernández-Pacheco, A.; Serrano-Ramón, L.; Michalik, J. M.; Ibarra, M. R.; De Teresa, J. M.; O’Brien, L.; Petit, D.; Lee, J.; Cowburn, R. P. Sci. Rep. 2013, 3, 1492. doi:10.1038/srep01492

19. Franken, J. H.; van der Heijden, M. A. J.; Ellis, T. H.; Lavrijsen, R.; Daniels, C.; McGrouther, D.; Swagten, H. J. M.; Koopmans, B. Adv. Funct. Mater. 2014, 24, 3508-3514. doi:10.1002/adfm.201303540

20. Vavassori, P.; Pancaldi, M.; Perez-Roldan, M. J.; Chuvilin, A.; Berger, A. Small 2016, 12, 1013-1023. doi:10.1002/smll.201503351

21. Lapicki, A.; Kang, K.; Suzuki, T. IEEE Trans. Magn. 2002, 38 , 2589-2591. doi:10.1109/TMAG.2002.803224

22. Ervin, M. H.; Nichols, B. M. J. Vac. Sci. Technol., B 2009, 27, 2982. doi:10.1116/1.3250259

23. Gavagnin, M.; Wanzenboeck, H. D.; Belic, D.; Shawrav, M. M.; Persson, A.; Gunnarsson, K.; Svedlindh, P.; Bertagnolli, E. Phys. Status Solidi A 2014, 211, 368-374. doi:10.1002/pssa.201330114

24. Tu, F.; Drost, M.; Vollnhals, F.; Späth, A.; Carrasco, E.; Fink, R. H.; Marbach, H. Nanotechnology 2016, 27, 355302. doi:10.1088/0957-4484/27/35/355302

25. Pohlit, M.; Porrati, F.; Huth, M.; Ohno, Y.; Ohno, H.; Müller, J. J. Appl. Phys. 2015, 117, 17C746. doi:10.1063/1.4917497

26. Fernández-Pacheco, A.; Serrano-Ramón, L. E.; Tyliszczak, T.; Chou, K. W.; Córdoba, R.; Szkudlarek, A.; Brien, L. O.; Kapusta, C.; Ibarra, M. R.; De Teresa, J. M. Nanotechnology 2012, 23, 105703. doi:10.1088/0957-4484/23/10/105703

27. Sangiao, S.; Morellón, L.; Ibarra, M. R.; De Teresa, J. M. Solid State Commun. 2011, 151, 37-41. doi:10.1016/j.ssc.2010.10.028

28. Fowlkes, J. D.; Winkler, R.; Lewis, B. B.; Stanford, M. G.; Plank, H.; Rack, P. D. ACS Nano 2016, 10, 6163-6172. doi:10.1021/acsnano.6b02108

29. Silvis-Cividjian, N.; Hagen, C. W.; Kruit, P. J. Appl. Phys. 2005, 98, 084905. doi:10.1063/1.2085307

30. Bernau, L.; Gabureac, M.; Erni, R.; Utke, I. Angew. Chem., Int. Ed. 2010, 49, 8880-8884. doi:10.1002/anie.201004220

31. Pablo-Navarro, J.; Sanz-Hernández, D.; Magén, C.; Fernández-Pacheco, A.; de Teresa, J. M. J. Phys. D: Appl. Phys. 2017, 50, 18LT01. doi:10.1088/1361-6463/aa63b4

32. Peinado, P.; Sangiao, S.; De Teresa, J. M. ACS Nano 2015, 9 , 6139-6146. doi:10.1021/acsnano.5b01383 
33. Fernández-Pacheco, A.; De Teresa, J. M.; Córdoba, R.; Ibarra, M. R. J. Phys. D: Appl. Phys. 2009, 42, 055005. doi:10.1088/0022-3727/42/5/055005

34. Córdoba, R.; Sharma, N.; Kölling, S.; Koenraad, P. M.; Koopmans, B. Nanotechnology 2016, 27, 355301. doi:10.1088/0957-4484/27/35/355301

35. Córdoba, R.; Sesé, J.; De Teresa, J. M.; Ibarra, M. R. Microelectron. Eng. 2010, 87, 1550-1553. doi:10.1016/j.mee.2009.11.027

36. Mulders, J. J. L.; Belova, L. M.; Riazanova, A. Nanotechnology 2010, 22, 055302. doi:10.1088/0957-4484/22/5/055302

37. Gazzadi, G. C.; Mulders, J. J. L.; Trompenaars, P.; Ghirri, A.; Rota, A.; Affronte, M.; Frabboni, S. Microelectron. Eng. 2011, 88, 1955-1958. doi:10.1016/j.mee.2010.12.031

38. Begun, E.; Dobrovolskiy, O. V.; Kompaniiets, M.; Sachser, R.; Gspan, C.; Plank, H.; Huth, M. Nanotechnology 2015, 26, 075301. doi:10.1088/0957-4484/26/7/075301

39. Puydinger dos Santos, M. V.; Velo, M. F.; Domingos, R. D.; Zhang, Y.; Maeder, X.; Guerra-Nuñez, C.; Best, J. P.; Béron, F.; Pirota, K. R.; Moshkalev, S.; Diniz, J. A.; Utke, I. ACS Appl. Mater. Interfaces 2016, 8, 32496-32503. doi:10.1021/acsami.6b12192

40. Schirmer, M.; Walz, M.-M.; Papp, C.; Kronast, F.; Gray, A. X.; Balke, B.; Cramm, S.; Fadley, C. S.; Steinrück, H.-P.; Marbach, H. Nanotechnology 2011, 22, 475304. doi:10.1088/0957-4484/22/47/475304

41. Sidles, J. A.; Garbini, J. L.; Bruland, K. J.; Rugar, D.; Züger, O.; Hoen, S.; Yannoni, C. S. Rev. Mod. Phys. 1995, 67, 249-265. doi:10.1103/RevModPhys.67.249

42. Degen, C. L.; Poggio, M.; Mamin, H. J.; Rettner, C. T.; Rugar, D. Proc. Natl. Acad. Sci. U. S. A. 2009, 106, 1313-1317. doi:10.1073/pnas.0812068106

43. Lee, I.; Obukhov, Y.; Xiang, G.; Hauser, A.; Yang, F.; Banerjee, P.; Pelekhov, D. V.; Hammel, P. C. Nature 2010, 466, 845-848. doi:10.1038/nature09279

44. Naletov, V. V.; de Loubens, G.; Albuquerque, G.; Borlenghi, S.; Cros, V.; Faini, G.; Grollier, J.; Hurdequint, H.; Locatelli, N.; Pigeau, B.; Slavin, A. N.; Tiberkevich, V. S.; Ulysse, C.; Valet, T.; Klein, O. Phys. Rev. B 2011, 84, 224423. doi:10.1103/PhysRevB.84.224423

45. Guo, F.; Belova, L. M.; McMichael, R. D. Phys. Rev. Lett. 2013, 110, 017601. doi:10.1103/PhysRevLett.110.017601

46. Mamin, H. J.; Rettner, C. T.; Sherwood, M. H.; Gao, L.; Rugar, D. Appl. Phys. Lett. 2012, 100, 013102. doi:10.1063/1.3673910

47. Mamin, H. J.; Rugar, D. Appl. Phys. Lett. 2001, 79, 3358-3360. doi:10.1063/1.1418256

48. Hickman, S. A.; Moore, E. W.; Lee, S.; Longenecker, J. G.; Wright, S. J.; Harrell, L. E.; Marohn, J. A. ACS Nano 2010, 4 7141-7150. doi:10.1021/nn101577t

49. Klein, O.; de Loubens, G.; Naletov, V. V.; Boust, F.; Guillet, T.; Hurdequint, H.; Leksikov, A.; Slavin, A. N.; Tiberkevich, V. S.; Vukadinovic, N. Phys. Rev. B 2008, 78, 144410. doi:10.1103/PhysRevB.78.144410

50. Pablo-Navarro, J.; Magén, C.; de Teresa, J. M. Nanotechnology 2016, 27, 285302. doi:10.1088/0957-4484/27/28/285302

51. Snoeck, E.; Gatel, C.; Lacroix, L. M.; Blon, T.; Lachaize, S.; Carrey, J.; Respaud, M.; Chaudret, B. Nano Lett. 2008, 8, 4293-4298. doi:10.1021/nl801998x

\section{License and Terms}

This is an Open Access article under the terms of the Creative Commons Attribution License (http://creativecommons.org/licenses/by/4.0), which permits unrestricted use, distribution, and reproduction in any medium, provided the original work is properly cited.

The license is subject to the Beilstein Journal of Nanotechnology terms and conditions: (http://www.beilstein-journals.org/bjnano)

The definitive version of this article is the electronic one which can be found at:

doi:10.3762/bjnano.8.210 